\title{
The clinical characteristics of Mycoplasma pneumoniae pneumonia and its relationship between hypokalemia in west China
}

\author{
Zhoubo Han", Yong Zhang", Shunli Liao, Na Zhou \\ Department of Pediatrics, The People's Hospital of Bishan District, Chongqing, China \\ Contributions: (I) Conception and design: N Zhou; (II) Administrative support: S Liao; (III) Provision of study materials or patients: Z Han, Y Zhang, \\ S Liao; (IV) Collection and assembly of data: Z Han, Y Zhang; (V) Data analysis and interpretation: N Zhou, Z Han, Y Zhang; (VI) Manuscript \\ writing: All authors; (VII) Final approval of manuscript: All authors. \\ "These authors contributed equally to this work. \\ Correspondence to: Na Zhou. Department of Pediatrics, The People's Hospital of Bishan District, No. 9, Shuangxing Road, Bishan District, \\ Chongqing 402760, China. Email: 519739790@qq.com.
}

Background: Mycoplasma pneumoniae (MP) is one of the most common respiratory pathogens of community acquired pneumonia (CAP) in children. The purpose of this study was to identify epidemics of Mycoplasma pneumoniae pneumonia (MPP) and to determine the relationship between hypokalemia and MPP.

Methods: This study was retrospectively conducted from 1 August 2016 to 31 July 2018 at Bishan People's Hospital in Chongqing, China. We analyzed the demographic characteristics (age, gender), extra-pulmonary manifestations, fever duration, length of hospital stay, whole blood cell (WBC) count, C-reactive protein (CRP) levels, and lactate dehydrogenase (LDH) levels.

Results: The total number of children with pneumonia was 3,385, including 570 (16.84\%) patients in the MP infection group. The male-to-female ratio was 1.34:1. The peak time of MPP incidence was in autumn. The MP infection group had a significantly higher ratio of fever, higher body temperature, and longer fever duration. The number of MP-infected patients with hypokalemia was highest in spring. The length of hospital stay in the mild hypokalemia group was longer than in the normokalemia group. Furthermore, severe hypokalemia cases were all in the severe MP infection group, and the severity of MP infection and hypokalemia were positively correlated.

Conclusions: In this study, we concluded that children infected with MP were more susceptible to fever, and had a longer duration of fever. The number of MP-infected patients with mild hypokalemia peaked in spring, and affected more boys than girls. Furthermore, patients with severe hypokalemia had longer fever duration, and a correlation was found between the severity of MP infection and hypokalemia.

Keywords: Mycoplasma pneumoniae pneumonia (MPP); fever; hypokalemia

Submitted Dec 02, 2020. Accepted for publication Jan 26, 2021.

doi: $10.21037 /$ tp-20-471

View this article at: http://dx.doi.org/10.21037/tp-20-471

\section{Introduction}

Mycoplasma pneumoniae (MP) is one of the most common respiratory pathogens of community acquired pneumonia (CAP), accounting for $20-40 \%$ of pneumonia in children $(1,2)$. Mycoplasma pneumoniae pneumonia (MPP) contributes to a broad array of pulmonary complications, such as pulmonary necrosis and chronic interstitial fibrosis (3), and has therefore attracted significant clinical attention. Recent evidence has shown that MP infection is also connected with the onset of several extra-pulmonary diseases, involving 
the skin, musculoskeletal, hematological digestive, nervous, and renal systems (4).

Potassium $\left(\mathrm{K}^{+}\right)$, the most abundant cation in humans, which is essential for normal cellular function. It can prevent myocardial failure through weakening cellular hyperpolarity and depolarization $(5,6)$. Hypokalemia is defined as a serum $\mathrm{K}^{+}$level of less than $3.5 \mathrm{mmol} / \mathrm{L}$, and hyperkalemia is defined as $\mathrm{K}^{+}$levels above $5.5 \mathrm{mmol} / \mathrm{L}$. A previous study reported that the degree of hypokalemia was associated with some clinical menifestations including high body temperature, and elevated laboratory indices reflecting myocardial injuries (7). Chen et al. (8) reported that patients with severe COVID-19 and severe hypokalemia $\left(\mathrm{K}^{+}\right.$levels below $3 \mathrm{mmol} / \mathrm{L}$ ) experienced respiratory muscle dysfunction and ventricular arrhythmia, both of which can be life-threatening.

There were no reports about the relationship between hypokalemia and MPP. We conducted this study to identify epidemics of MPP in west China, and to explore the relationship between hypokalemia and MPP.

We present the following article in accordance with the STROBE reporting checklist (available at http://dx.doi. org/10.21037/tp-20-471).

\section{Methods}

\section{Study population}

This study was retrospectively conducted at the Bishan People's Hospital in Chongqing, China. Patients included hospitalized children with MPP, without vomiting or diarrhea. Data from patients were analyzed anonymously between 1 August 2016 and 31 July 2018.

\section{Definitions}

MPP was diagnosed according to the following criteria: (I) clinical presentation (fever, cough), (II) chest imaging with pulmonary infiltrates, (III) positive results for MP with serum anti-MP IgM titer $\geq 1: 160$.

Children with any of the following factors were excluded: (I) neonatal pneumonia, (II) diarrhea, which was defined as the passage of 3 or more abnormally loose or watery stools in the previous 24 hours (9), (III) convalescent pneumonia, (IV) long-term use of glucocorticoids or immunosuppressive agents, and $(\mathrm{V})$ congenital heart or chronic lung disease.

\section{Ethics statement}

The study was conducted in accordance with the guiding principles of the Declaration of Helsinki (as revised in 2013), and the study approach was approved by the Ethical Review Committee of Bishan People's Hospital. This study only collected the clinical data of patients, did not interfere with the treatment plan of patients, and did not bring physiological risks to patients. Because of the retrospective nature of the study, patient consent for inclusion was waived. We did our best to protect the privacy of personal information provided by patients.

\section{Clinical data}

Medical records of the study population were retrospectively reviewed. We collected the records including demographic characteristics (age, gender), extra-pulmonary manifestations, fever duration, and length of hospital stay from all children included in the study. The data were registered by our own pediatricians. We finally used months as a unit of age.

\section{Laboratory data}

Peripheral blood samples were obtained on admission for the determination of WBC, CRP, LDH, and specific antibody to MP. All the examinations were completed in accordance with the standard protocol of the laboratory department. The report results were reviewed by 2 different medical lab staff members and released to the patients.

\section{Statistical analysis}

Continuous variables were presented as medians with $25 \%$ and $75 \%$ quartiles (interquartile range, IQR), and the non-parametric test and Mann-Whitney $U$ test were used for analysis. Categorical variables were presented as counts (percentages), and assessed using the Kruskal-Wallis test or Fisher's exact test. Correlations between variables were analyzed using Pearson's correlation coefficient. Binary logistic regression tests were used to evaluate the association between the severity of MP infection and potassium. Statistical analyses were conducted using SPSS 25.0. 
Table 1 Characteristics of children with pneumonia from 1 August 2016 to 31 July 2018

\begin{tabular}{|c|c|c|c|}
\hline Item & No MP infection & MP infection & $P$ \\
\hline Sex (male), n (\%) & $1,622(57.60)$ & $318(57.80)$ & 0.420 \\
\hline Age (months), median [IQR] & 24 [10-49] & $22[9-46]$ & 0.143 \\
\hline Season at enrollment, n (\%) & & & 0.002 \\
\hline Summer (June-August) & $575(20.40)$ & $83(14.60)$ & \\
\hline Autumn (September-November) & $731(26.00)$ & $181(31.80)$ & \\
\hline Winter (December-February) & $758(26.90)$ & $158(27.70)$ & \\
\hline Heart rate $[\mathrm{bpm}]$, median & $121[115-127]$ & 116 [109-122] & 0.000 \\
\hline Max temperature $\left({ }^{\circ} \mathrm{C}\right)$, median & 39 (38.5-39.5) & $39.2(38.7-39.5)$ & 0.001 \\
\hline Fever duration [day], median & $4[2-5]$ & $4[2-6]$ & 0.000 \\
\hline Wheezing, n (\%) & $585(20.80)$ & $92(16.10)$ & 0.012 \\
\hline Length of stay [day], median & $5[4-6]$ & $5[4-6]$ & 0.599 \\
\hline WBC count $\left(\times 10^{9} / \mathrm{L}\right)$, median $(\mathrm{IQR})$ & $9.83(7.32-13.80)$ & $9.26(6.96-12.19)$ & 0.017 \\
\hline Ratio of neutrophils (\%), median (IQR) & $47(32.60-61.60)$ & $57.7(44.30-67.90)$ & 0.000 \\
\hline CRP (mg/L), median (IQR) & $2.64(0-9.71)$ & $3.74(0-9.97)$ & 0.303 \\
\hline LDH (U/L), median [IQR] & 281 [245-322] & 261 [227-294] & 0.000 \\
\hline
\end{tabular}

MP, Mycoplasma pneumoniae; IQR, interquartile range; WBC, whole blood cell; CRP, C-reactive protein; LDH, lactate dehydrogenase.

\section{Results}

\section{Characteristics of children with pneumonia}

As shown in Table 1, the total number of children with pneumonia was 3,385 , including the no MP infection group with $2,815(83.16 \%)$ patients and the MP infection group with $570(16.84 \%)$ patients. Age ranged from 1 month to 178 months, with a mean age of $32.7 \pm 29.88$ months. The male-to-female ratio was 1.34:1. Cases were found more frequently during spring, autumn, and winter: from March to May in 899 cases (26.6\%), from June to August in 658 cases (19.4\%), from September to November in 912 cases (26.9\%), and from December to February in 916 cases (27.1\%).

\section{Age and gender distribution}

The median age of the MP infection group (22 months,
IQR, 9-46 months) was similar to that of the no MP infection group (24 months, IQR, 10-49 months, $\mathrm{P}=0.143$ ). There were 1,622 boys in the no MP infection group (57.6\%) and 318 boys in the MP infection group (57.8\%, $\mathrm{P}=0.143)$.

\section{Seasonal distribution}

The no MP infection group and the MP infection group had 751 cases $(26.7 \%)$ and 148 cases $(26.0 \%)$ in spring, 575 cases $(20.4 \%)$ and 83 cases $(14.6 \%)$ in summer, 731 cases $(26.0 \%)$ and 181 cases $(31.8 \%)$ in autumn, and 758 cases $(26.9 \%)$ and 158 cases $(27.7 \%)$ in winter, respectively $(\mathrm{P}=0.000)$. The number of cases between the groups was significantly different, especially in summer and autumn $(\mathrm{P}=0.000)$. In addition, we found that the peak time of MPP incidence was in autumn. 


\section{Clinical symptoms}

The clinical characteristics of the no MP infection group and the MP infection group are shown in Table 1. Among the children with MP, the most common respiratory symptoms were fever and wheeze, prompting us to compare the clinical conditions of the 2 groups.

The MP infection group had a significantly higher ratio of fever $(398 / 570,69.8 \%)$ than that of the no MP infection group $(1,743 / 2,815,61.9 \%, \mathrm{P}=0.000)$. Furthermore, the MP infection group had higher body temperature (median $\left.39.2{ }^{\circ} \mathrm{C}, \mathrm{IQR}, 38.7-39.5{ }^{\circ} \mathrm{C}, \mathrm{P}=0.001\right)$ and longer fever duration (median 4 days, IQR, 4-6 days, $\mathrm{P}=0.000$ ).

Our study also showed that the MP infection group had a significantly lower ratio of wheeze $(92 / 570,16.1 \%)$ than that of the no MP infection group $(585 / 2,815,20.8 \%$, $\mathrm{P}=0.012)$. In addition, the MP infection group had a lower heart rate (116 vs. $121 \mathrm{bpm}, \mathrm{P}=0.000)$ and a lower respiratory rate $(28 v s .32 \mathrm{bpm}, \mathrm{P}=0.000)$ compared to the no MP infection group.

\section{Laboratory tests}

The whole blood cell (WBC) count of the MP infection group (median $9.26 \times 10^{9} / \mathrm{L}$, IQR, $6.96-12.19 \times 10^{9} / \mathrm{L}$ ) was lower than that of the no MP infection group (median $9.83 \times 10^{9} / \mathrm{L}, \mathrm{IQR}, 7.32-13.80 \times 10^{9} / \mathrm{L}, \mathrm{P}=0.017$ ). However, the ratio of neutrophils was higher in the MP infection group than in the no MP infection group $(\mathrm{P}=0.000)$. CRP was higher in the MP infection group (median $3.74 \mathrm{mg} / \mathrm{L}$, IQR, $0-9.97 \mathrm{mg} / \mathrm{L}$ ) than in the no MP infection group (median $2.64 \mathrm{mg} / \mathrm{L}, \mathrm{IQR}, 0-9.71 \mathrm{mg} / \mathrm{L})(\mathrm{P}=0.303)$, while both groups were within the normal reference range.

\section{MPP and potassium}

In order to study the effect of potassium on MP, especially the effect of hypokalemia, we divided the patients with confirmed MPP into 4 groups: normokalemia group, mild hypokalemia group, severe hypokalemia group, and hyperkalemia group. The specific data are presented in Table 2. Out of the 570 patients with MP in this study, the 4 groups had 489, 73, 4, and 4 patients, respectively.

We found that there were no female patients with hypokalemia, but there were 73 male patients with mild hypokalemia and 4 male patients with severe hypokalemia $(\mathrm{P}=0.000)$. There were $148,83,181$, and 158 patients with MP infections across the 4 seasons, respectively. In order to determine whether season had an effect on potassium, we found that there were 105 cases $(21.5 \%)$ and 41 cases $(56.2 \%)$ in spring, 70 cases $(14.30 \%)$ and 13 cases $(17.8 \%)$ in summer, 177 cases $(36.2 \%)$ and 1 case (1.4\%) in autumn, and 137 cases (28\%) and 18 cases (24.7\%) in the normokalemia group and the mild hypokalemia group, respectively. The differences between groups across the seasons were statistically significant $(\mathrm{P}=0.000)$. Furthermore, there were more normokalemia cases in autumn $(36.2 \%)$, but more cases with mild hypokalemia in spring (56.2\%), and the difference between the normokalemia group and the mild hypokalemia group was statistically significant $(\mathrm{P}=0.000)$. All these data demonstrated that the number of patients with MP infection and hypokalemia was higher in spring. After analyzing the age distribution, we found that the age of the mild hypokalemia group (median 17 months, IQR, 2.75-20 months) was lower than that of the normokalemia group (median 25 months, IQR, 1047 months, $\mathrm{P}=0.007$ ). This finding indicated that patients with mild hypokalemia were younger than patients in the normokalemia group.

The length of hospital stay in the mild hypokalemia group (median 6 days, IQR, 6-7 days) was longer than that of the normokalemia group (median 5 days, IQR, 4-5 days, $\mathrm{P}=0.000)$.

There were no differences in the ratio of fever, maximum temperature, and the duration of fever among the 4 groups. However, among the fever cases, the severe hypokalemia group had a longer fever duration (median 8 days) compared to the normokalemia group (median 4 days, IQR, $2-6, \mathrm{P}=0.037$ ) and the mild hypokalemia group (median 4 days, IQR, 3-5.5 days, $\mathrm{P}=0.035$ ). The results demonstrated that patients with severe hypokalemia had a longer fever duration.

Results also demonstrated that there were no marked differences in WBC, ratio of neutrophils, CRP, and LDH among the 4 groups.

In order to determine the relationship between hypokalemia and the titer of serum anti-MP IgM, we found that across the 4 groups, there were 477 cases $(86.3 \%), 72$ cases $(13 \%), 0$ cases $(0 \%)$, and 4 cases $(0.7 \%)$ in the mild MP infection group, respectively, and 412 cases (70.6\%), 1 case $(1.4 \%), 4$ cases $(23.5 \%)$, and 0 cases $(0 \%)$ in the severe MP infection group, respectively. The differences were statistically significant $(\mathrm{P}=0.000)$. Furthermore, the severe hypokalemia patients were all in the severe MP infection group, and logistic regression analysis also showed that the severity of MP infection and potassium levels were 
Table 2 The characteristics of MPP with hyperkalemia and different levels of hypokalemia

\begin{tabular}{|c|c|c|c|c|c|}
\hline Item & Normokalemia group & $\begin{array}{l}\text { Mild hypokalemia } \\
\text { group }\end{array}$ & $\begin{array}{l}\text { Severe hypokalemia } \\
\text { group }\end{array}$ & Hyperkalemia group & $P$ \\
\hline Case, $\mathrm{n}$ & 489 & 73 & 4 & 4 & \\
\hline Male, $\mathrm{n}$ & 238 & 73 & 4 & 3 & 0.000 \\
\hline Season at enrollment, n (\%) & & & & & 0.000 \\
\hline Spring (March-May) & $105(21.5)$ & $41(56.2)^{\circledR}$ & $2(50.0)$ & 0 & \\
\hline Summer (June-August) & $70(14.3)$ & $13(17.8)$ & 0 & 0 & \\
\hline Respiratory rate $[\mathrm{bpm}]$, median & 28 [25-32] & 28 [25-32] & 29 [24-33] & 28 [22-34] & 0.972 \\
\hline Fever, n (\%) & 339 & 54 & 3 & 2 & 0.694 \\
\hline Max temperature $\left({ }^{\circ} \mathrm{C}\right)$, median & $39.2(38.7-39.5)$ & $39(38.4-39.5)$ & 38.5 & 39.3 & \\
\hline Fever duration [day], median & $4[2-6]$ & $4[3-5.5]$ & $8^{\#}$ & 5.5 & \\
\hline Wheezing, n (\%) & 81 & 9 & 1 & 1 & 0.726 \\
\hline Length of stay (day), median & 5 & 6 & 6 & 3.5 & 0.000 \\
\hline WBC count $\left(\times 10^{9} / \mathrm{L}\right)$, median $(\mathrm{IQR})$ & $9.32(6.98-12.46)$ & $8.60(6.82-11.34)$ & $8.97(4.90-12.45)$ & $8.86(6.01-17.96)$ & 0.818 \\
\hline $\begin{array}{l}\text { Severe MP infection (serum anti-MP } \\
\text { lgM titer } \geq 1: 320), n(\%)\end{array}$ & 12 (70.6) & $1(1.4)$ & $4(23.5)$ & $0(0.0)$ & \\
\hline
\end{tabular}

correlated $(\mathrm{r}=0.98, \mathrm{P}=0.002)$.

\section{Discussion}

In our study, the incidence of MP was $16.84 \%$ and the male-to-female ratio was $1.34: 1$, which was generally consistent with the reported data (10). Seasonal forcing has been proposed as a determinant for the periodic patterns in some infectious diseases. Our results suggested that MPP occurred all year round, but the incidence was lowest in summer and highest in autumn. This is different from some previous reports, including a study by Waites et al. (11) who found that MP infections tended to be more common in summer or early autumn. Previous results have also shown that elements of the local climate such as higher temperature and relative humidity can influence MPP incidence (12-14). Hence, the distributions of MP may vary across the year, and this may be different among different 
cities and countries.

Notably, our study revealed that the pattern of positive detections stratified by age. With detection in all age groups, there was no difference among groups in our study. This is in contrast to a previous report showing that MP cases were classically observed among pre-school or schoolaged children $(11,15)$. However, another study showed that $39.2 \%$ of children aged from 0-4 years were diagnosed with MMP infection (12). IgM antibodies are used for the diagnosis of MP infection, though elevated $\operatorname{IgM}$ will decay slowly up to 6 months. Thus, doctors are not sure whether the reaction is from current infection or from a primary abnormality. In our study, we also used IgM antibodies for the diagnosis of MP infection, but the median age of the MP infection group was 22 months (IQR, 9-46 months), which was school age. Hence, we believed the IgM test was the most efficient way to make a serological diagnosis in a single laboratory examination compared to other diagnostic tests.

Among children with MP infection, the most common respiratory symptoms were cough, dyspnea, fever, and wheeze. Fever is a common clinical symptom in children and is one of the leading causes for medical consultation and hospital admissions (16). In our study, we found that the patients with MP infection (51.5\%) had a higher ratio of fever than those with no MP infection $(11.7 \%, \mathrm{P}=0.000)$. At the same time, patients with MP infection also had a longer duration of fever. We inferred that patients with MP infection were more susceptible to fever. Therefore, even MP is a self-limited disease, and can cause a higher ratio of fever and a longer duration of fever, making these children less comfortable, warranting more attention to this problem. Reports have suggested that MP plays a role in acute episodes of wheezing, and is also associated with the development of asthma $(14,17)$. However, in our study, patients with MP infection $(2.7 \%)$ had a lower ratio of wheeze than those with no MP infection (17.3\%, $\mathrm{P}=0.011)$. The median age of the MP infection group and the no MP infection group were 22 and 24 months, respectively, which was in early childhood. It is known that viruses are the main cause of wheeze during this period (2).

As many as $25 \%$ of people infected with MP may experience extra-pulmonary complications (18), and MPrelated extra-pulmonary diseases represent the most evident clinical problem of this infection, especially in children (19). MP can adhere to other cells, such as red blood cells and macrophages (14). In our study, we found that the WBC count was lower in the MP infection group than in the no MP infection group ( $\mathrm{P}=0.034)$, but the ratio of neutrophils and CRP was higher in the MP infection group compared to the no MP infection group $(\mathrm{P}=0.000$, $\mathrm{P}=0.312$ ). This result was in agreement with previous data, which showed that the WBC count was mostly normal or slightly elevated, and neutrophils were found by the differential counts in the cases of MP infection. Stevens and Krause (20) also observed that $64 \%$ of cases had normal WBC count. Furthermore, Gao et al. (21) showed that children with positive MP serology had higher CRP levels. Therefore, in our clinical work, if routine blood examination shows that the WBC count is at a normal or slightly elevated level and the ratio of neutrophils and CRP is higher, we can conclude that the patient may have MP infection. $\mathrm{LDH}$ is released from cells after cell damage, and the LDH level can reflect the degree of cell and tissue damage. In this study, the levels of LDH were higher in the no MP infection group than in the MP infection group, but all were within the normal range. A higher level of LDH can translate into excessive inflammatory cell infiltration and severe lung injury, which is indicative of refractory MPP (RMPP). Therefore, serum LDH level has been considered a marker for RMPP (22). However, in our study, there were few cases of RMPP, suggesting that for cases with no RMPP, LDH may have little clinical significance.

Hyponatremia, hypokalemia, and hypocalcemia have been reported in sick children. Hypokalemia gives rise to cellular hyperpolarity, increases resting potential, and hastens depolarization in cardiac cells and lung cells. Severe hypokalemia can result in ventricular arrhythmia and respiratory muscle dysfunction, which are life-threatening conditions in patients $(8,23)$. More research and education about dyskalemia may help increase awareness about the issue and improve clinical practice. Our study found that patients with MP infection and hypokalemia were all males, and there was a strong association between gender and hypokalemia in MP-infected patients. In addition, patients with MP infection and hypokalemia had a longer length of hospital stay and the patients with severe hypokalemia had a longer duration of fever. This finding has never been reported, but we regret for the limit of the small sample scale. We also found that the cases of severe hypokalemia were all in the severe MP infection group, and logistic regression analysis showed that the severity of MP infection and potassium were negatively correlated. Therefore, patients with a higher titer of serum anti-MP IgM were more likely to be hypokalemic. We advise that children 
with pneumonia who also have hypokalemia should receive closer observation at health care facilities in clinical practice. Hypokalemia is common in sick and hospitalized patients, but the severity can vary (23). Potassium content and its distribution rely on a complex interplay of multiple factors, including renal and gastrointestinal function, and diet (5). Findings have also shown a strong association between diarrhea and hypokalemia, as there may be a further reduction in potassium levels due to loss though diarrheal stools (24). However, in our study, we excluded patients with diarrhea and chronic diseases to eliminate interference.

Hyperkalemia $(>5.5 \mathrm{mmol} / \mathrm{L}$ ) has been associated with an increased risk of death, even when potassium levels reached $6.0 \mathrm{mmol} / \mathrm{L}$ (5). In this study, there were few patients with hyperkalemia, which might have impacted the level of statistical significance for some of the variables among groups. Based on these results, we propose that there may be some association between these factors, but further study is needed.

$\mathrm{MP}$ is one of the most common respiratory pathogens of CAP in children, it is of great significance to study it in depth. What our group can do is to last the study time and increase the sample size to find more interesting phenomenon.

In summary, our results indicated that children with MP infection were more susceptible to fever and had a longer fever duration, while patients with severe hypokalemia also had a longer duration of fever. We also found that the number of MP-infected patients with mild hypokalemia peaked in spring, affecting more boys than girls. Furthermore, a positive correlation was found between the severity of MP infection and hypokalemia.

\section{Limitations}

This study has a few limitations. Firstly, our study was performed prospectively using IgM antibodies for the diagnosis of MP infection. Methodologies for the detection of MP include nucleic acid amplification tests (NAAT), serology, and culture. But there are no internationally defined guidelines on MP (25). There are some limitations of the $\operatorname{IgM}$ test, but compared to other diagnostic tests, serological diagnosis is the most efficient in a single laboratory examination. Secondly, the severity of MP infection has there are several risk factors. The confounding factors might affect the results. However, as the present findings reflect the fundamental pathophysiological mechanisms of MP infection and the large proportion of patients with hypokalemia, we believe that our conclusions are valid. Lastly, the small sample size is the main limitation of our study, especially when the patients were grouped by potassium concentration. In future studies, more data using a larger sample size should be collected.

\section{Acknowledgments}

Funding: The authors disclosed receipt of the following financial support for the research, authorship, and/or publication of this article: This work was funded by the Medical research project of Chongqing health commission (No. 2015MSXM184).

\section{Footnote}

Reporting Checklist: The authors have completed the STROBE reporting checklist. Available at http://dx.doi. org/10.21037/tp-20-471

Data Sharing Statement: Available at http://dx.doi. org/10.21037/tp-20-471

Conflicts of Interest: All authors have completed the ICMJE uniform disclosure form (available at http://dx.doi. org/10.21037/tp-20-471). All authors report grants from Medical research project of Chongqing health commission (No. 2015MSXM184) during the conduct of the study. The authors have no other conflicts of interest to declare.

Ethical Statement: The authors are accountable for all aspects of the work in ensuring that questions related to the accuracy or integrity of any part of the work are appropriately investigated and resolved. The study was conducted in accordance with the principles of the Declaration of Helsinki (as revised in 2013), and the study protocol was approved by the Ethical Review Committee of Bishan People's Hospital. This study only collected the clinical data of patients, did not interfere with the treatment plan of patients, and did not bring physiological risks to patients. Because of the retrospective nature of the study, patient consent for inclusion was waived. We did our best to protect the privacy of personal information provided by patients.

Open Access Statement: This is an Open Access article 
distributed in accordance with the Creative Commons Attribution-NonCommercial-NoDerivs 4.0 International License (CC BY-NC-ND 4.0), which permits the noncommercial replication and distribution of the article with the strict proviso that no changes or edits are made and the original work is properly cited (including links to both the formal publication through the relevant DOI and the license). See: https://creativecommons.org/licenses/by-nc$\mathrm{nd} / 4.0 /$.

\section{References}

1. Atkinson TP, Balish MF, Waites KB. Epidemiology, clinical manifestations, pathogenesis and laboratory detection of Mycoplasma pneumoniae infections. FEMS Microbiol Rev 2008;32:956-73.

2. Han MS, Yun KW, Lee HJ, et al. Contribution of Codetected Respiratory Viruses and Patient Age to the Clinical Manifestations of Mycoplasma Pneumoniae Pneumonia in Children. Pediatr Infect Dis J 2018;37:531-6.

3. Sawicki GS, Lu FL, Valim C, et al. Necrotising pneumonia is an increasingly detected complication of pneumonia in children. Eur Respir J 2008;31:1285-91.

4. Xiao L, Ratliff AE, Crabb DM, et al. Molecular Characterization of Mycoplasma pneumoniae Isolates in the United States from 2012 to 2018. J Clin Microbiol 2020;58:e00710-20.

5. Ferreira JP, Butler J, Rossignol P, et al. Abnormalities of Potassium in Heart Failure: JACC State-of-the-Art Review. J Am Coll Cardiol 2020;75:2836-50.

6. Coca SG, Perazella MA, Buller GK. The cardiovascular implications of hypokalemia. Am J Kidney Dis 2005;45:233-47.

7. Chisti MJ, Salam MA, Ashraf H, et al. Predictors and outcome of hypoxemia in severely malnourished children under five with pneumonia: a case control design. PLoS One 2013;8:e51376.

8. Chen D, Li X, Song Q, et al. Assessment of Hypokalemia and Clinical Characteristics in Patients With Coronavirus Disease 2019 in Wenzhou, China. JAMA Netw Open 2020;3:e2011122.

9. Chisti MJ, Saha S, Roy CN, et al. Predictors of bacteremia in infants with diarrhea and systemic inflammatory response syndrome attending an urban diarrheal treatment center in a developing country*. Pediatric Critical Care Medicine 2010;11:92-7.

10. Zhang XS, Zhao H, Vynnycky E, et al. Positively interacting strains that co-circulate within a network structured population induce cycling epidemics of Mycoplasma pneumoniae. Sci Rep 2019;9:541.

11. Waites KB, Xiao L, Liu Y, et al. Mycoplasma pneumoniae from the Respiratory Tract and Beyond. Clin Microbiol Rev 2017;30:747-809.

12. Onozuka D, Chaves LF. Climate variability and nonstationary dynamics of Mycoplasma pneumoniae pneumonia in Japan. PLoS One 2014;9:e95447.

13. Onozuka D, Hashizume M, Hagihara A. Impact of weather factors on Mycoplasma pneumoniae pneumonia. Thorax 2009;64:507-11.

14. Waites KB, Talkington DF. Mycoplasma pneumoniae and its role as a human pathogen. Clin Microbiol Rev 2004;17:697-728, table of contents.

15. Kutty PK, Jain S, Taylor TH, et al. Mycoplasma pneumoniae Among Children Hospitalized With Community-acquired Pneumonia. Clin Infect Dis 2019;68:5-12.

16. Reiner RC, Olsen HE, Ikeda CT, et al. Diseases, Injuries, and Risk Factors in Child and Adolescent Health, 1990 to 2017. JAMA Pediatrics 2019;173:e190337.

17. Yeh JJ, Wang YC, Hsu WH, et al. Incident asthma and Mycoplasma pneumoniae: A nationwide cohort study. J Allergy Clin Immunol 2016;137:1017-23.e6.

18. Poddighe D. Extra-pulmonary diseases related to Mycoplasma pneumoniae in children: recent insights into the pathogenesis. Curr Opin Rheumatol 2018;30:380-7.

19. de Groot RCA, Meyer Sauteur PM, Unger WWJ, et al. Things that could be Mycoplasma pneumoniae. J Infect 2017;74:S95-S100.

20. Stevens MK, Krause DC. Mycoplasma pneumoniae cytadherence phase-variable protein HMW3 is a component of the attachment organelle. J Bacteriol 1992;174:4265-74.

21. Gao J, Yue B, Li H, et al. Epidemiology and clinical features of segmental/lobar pattern Mycoplasma pneumoniae pneumonia: A ten-year retrospective clinical study. Exp Ther Med 2015;10:2337-44.

22. Miyashita N, Kawai Y, Inamura N, et al. Setting a standard for the initiation of steroid therapy in refractory or severe Mycoplasma pneumoniae pneumonia in adolescents and adults. J Infect Chemother 2015;21:153-60.

23. Chen H, Ma R, Du H, et al. Early onset children's Gitelman syndrome with severe hypokalaemia: a case report. BMC Pediatr 2020;20:366.

24. Chisti MJ, Salam MA, Ashraf H, et al. Clinical signs of radiologic pneumonia in under-five hypokalemic diarrheal 
children admitted to an urban hospital in bangladesh.

PLoS One 2013;8:e71911.

25. Beeton ML, Zhang XS, Uldum SA, et al. Mycoplasma pneumoniae infections, 11 countries in Europe and Israel, 2011 to 2016. Euro Surveill 2020;25:1900112.

Cite this article as: Han Z, Zhang Y, Liao S, Zhou N. The clinical characteristics of Mycoplasma pneumoniae pneumonia and its relationship between hypokalemia in west China. Transl Pediatr 2021;10(2):406-414. doi: 10.21037/tp-20-471 